\title{
Linear Quadratic Nonzero Sum Differential Games with Asymmetric Information
}

\author{
Dejian Chang ${ }^{1,2}$ and Hua Xiao ${ }^{3}$ \\ ${ }^{1}$ Qilu Securities Institute for Financial Studies, Shandong University, Jinan 250100, China \\ ${ }^{2}$ School of Mathematics, Shandong University, Jinan 250100, China \\ ${ }^{3}$ School of Mathematics and Statistics, Shandong University, Weihai 264209, China
}

Correspondence should be addressed to Hua Xiao; xiao_hua@sdu.edu.cn

Received 3 March 2014; Accepted 7 July 2014; Published 5 August 2014

Academic Editor: Ming Gao

Copyright (C) 2014 D. Chang and H. Xiao. This is an open access article distributed under the Creative Commons Attribution License, which permits unrestricted use, distribution, and reproduction in any medium, provided the original work is properly cited.

\begin{abstract}
This paper studies a linear quadratic nonzero sum differential game problem with asymmetric information. Compared with the existing literature, a distinct feature is that the information available to players is asymmetric. Nash equilibrium points are obtained for several classes of asymmetric information by stochastic maximum principle and technique of completion square. The systems of some Riccati equations and forward-backward stochastic filtering equations are introduced and the existence and uniqueness of the solutions are proved. Finally, the unique Nash equilibrium point for each class of asymmetric information is represented in a feedback form of the optimal filtering of the state, through the solutions of the Riccati equations.
\end{abstract}

\section{Introduction}

Throughout this article, we denote by $R^{k}$ the $k$-dimensional Euclidean space, $R^{k \times l}$ the collection of $k \times l$ matrices. The superscript $*$ denotes the transpose of vectors or matrices. Let $\left(\Omega, \mathscr{F},\left(\mathscr{F}_{t}\right), P\right)$ be a complete filtered probability space in which $\mathscr{F}_{t}$ denotes a natural filtration generated by a three dimensional standard Brownian motion $\left(W_{1}(t), W_{2}(t), W_{3}(t)\right), \mathscr{F}=\mathscr{F}_{T}$, and $T>0$ be a fixed time horizon. For a given Euclidean space, we denote by $\langle\cdot, \cdot\rangle$ (resp., $|\cdot|)$ the scalar product (resp., norm). We also denote by $\mathscr{L}_{\mathscr{F}_{t}}^{2}(0, T ; S)$ the space of all $S$-valued, $\mathscr{F}_{t}$-adapted and square integrable processes, by $\mathscr{L}_{\mathscr{F}_{T}}^{2}(\Omega ; S)$ the space of all $S$-valued, $\mathscr{F}_{T}$-measurable and square integrable random variables, by $\mathscr{L}^{2}(0, T ; S)$ the space of all $S$-valued functions satisfying $\int_{0}^{T}|f(t)|^{2} d t<\infty$, and by $f(t)^{2}$ the square of $f(t)$. For the sake of simplicity, we set

$$
\mathscr{F}_{t}^{j}=\sigma\left\{W_{j}(s), 0 \leq s \leq t\right\} \quad(j=1,2,3),
$$

$$
\begin{gathered}
\mathscr{F}_{t}^{1,2}=\sigma\left\{W_{1}(s), W_{2}(s), 0 \leq s \leq t\right\}, \\
\mathscr{F}_{t}^{2,3}=\sigma\left\{W_{2}(s), W_{3}(s), 0 \leq s \leq t\right\}, \\
\widehat{h}(t)=\mathbb{E}\left(h(t) \mid \mathscr{F}_{t}^{1,2}\right), \\
\widetilde{h}(t)=\mathbb{E}\left(h(t) \mid \mathscr{F}_{t}^{2}\right), \quad \check{h}(t)=\mathbb{E}\left(h(t) \mid \mathscr{F}_{t}^{3}\right), \\
\bar{h}(t)=\mathbb{E}\left(h(t) \mid \mathscr{F}_{t}^{2,3}\right), \quad \dot{h}(t)=\frac{d h(t)}{d t} .
\end{gathered}
$$

This work is interested in linear quadratic (LQ, for short) non-zero sum differential game with asymmetric information. For simplicity, we only study the case of two players. Let us now begin to specify the problem. Consider the following one-dimensional stochastic differential equation (SDE, for short)

$$
\begin{aligned}
d x^{v_{1}, v_{2}}(t)=\left[a(t) x^{v_{1}, v_{2}}(t)+b_{1}(t) v_{1}(t)\right. & \\
& \left.+b_{2}(t) v_{2}(t)+c(t)\right] d t+g_{1}(t) d W_{1}(t)
\end{aligned}
$$




$$
\begin{aligned}
& +\left[e(t) x^{v_{1}, v_{2}}(t)+g_{2}(t)\right] d W_{2}(t) \\
& +g_{3}(t) d W_{3}(t), \\
& x^{v_{1}, v_{2}}(0)=x_{0},
\end{aligned}
$$

and cost functionals of the form

$$
\begin{gathered}
J_{i}\left(v_{1}(\cdot), v_{2}(\cdot)\right)=\frac{1}{2} \mathbb{E}\left[\int_{0}^{T}\left(l_{i}(t) x^{v_{1}, v_{2}}(t)^{2}+m_{i}(t) v_{i}(t)^{2}\right) d t\right. \\
\left.+r_{i} x^{v_{1}, v_{2}}(T)^{2}\right] \quad(i=1,2) .
\end{gathered}
$$

Here $a, b_{1}, b_{2}, c, e, g_{1}, g_{2}$ and $g_{3}$ are bounded and deterministic functions in $t, l_{1}$ and $l_{2}$ are bounded, nonnegative and deterministic functions in $t, m_{1}$ and $m_{2}$ are bounded, positive and deterministic functions in $t$, and $r_{1}$ and $r_{2}$ are two nonnegative constants. Hereinafter, we omit all dependence on time variable $t$ of all processes or deterministic functions if there is no risk of ambiguity from the context for the notational simplicity; $v_{1}(\cdot)$ and $v_{2}(\cdot)$ are the control processes of Player 1 and Player 2, respectively. We always use the subscript 1 (resp., the subscript 2) to characterize the control variable corresponding to Player 1 (resp., Player 2) and use the notation $x^{\nu_{1}, v_{2}}$ to denote the dependence of the state on the control variable $\left(v_{1}, v_{2}\right)$.

Let $\mathscr{F}_{t}$ denote the full information up to time $t$ and $\mathscr{G}_{t}^{i} \subseteq$ $\mathscr{F}_{t}$ be a given sub-filtration which represents the information available to Player $i(i=1,2)$ at time $t \in[0, T]$. If $\mathscr{G}_{t}^{i} \subseteq \mathscr{F}_{t}$ and $\mathscr{G}_{t}^{i} \neq \mathscr{F}_{t}$, we call the available information partial or incomplete for Player $i$. If $\mathscr{G}_{t}^{1} \neq \mathscr{G}_{t}^{2}$, we call the available information asymmetric for Player 1 and Player 2. Now we introduce the admissible control set

$$
\mathscr{U}_{i}=\left\{v_{i}(\cdot) \in \mathscr{L}_{\mathscr{G}_{t}^{i}}^{2}(0, T ; R) \mid v_{i}(t) \in U_{i}, t \in[0, T]\right\},
$$

where $\mathscr{G}^{i}=\mathscr{G}_{T}^{i}$ and $U_{i}$ are nonempty convex subsets of $R(i=$ $1,2)$. Each element of $\mathscr{U}_{i}$ is called an open-loop admissible control for Player $i(i=1,2)$. And $\mathscr{U}_{1} \times \mathscr{U}_{2}$ is said to be the set of open-loop admissible controls for the players.

Suppose each player hopes to minimize her/his cost functional $J_{i}\left(v_{1}(\cdot), v_{2}(\cdot)\right)$ by selecting a suitable admissible control $v_{i}(\cdot)(i=1,2)$. In this study, the problem is, under the setting of asymmetric information, to look for $\left(u_{1}(\cdot), u_{2}(\cdot)\right) \in$ $\mathcal{U}_{1} \times \mathcal{U}_{2}$ which is called the Nash equilibrium point of the game, such that

$$
\begin{aligned}
& J_{1}\left(u_{1}(\cdot), u_{2}(\cdot)\right)=\min _{v_{1}(\cdot) \in \mathscr{U}_{1}} J_{1}\left(v_{1}(\cdot), u_{2}(\cdot)\right), \\
& J_{2}\left(u_{1}(\cdot), u_{2}(\cdot)\right)=\min _{v_{2}(\cdot) \in \mathscr{U}_{2}} J_{1}\left(u_{1}(\cdot), v_{2}(\cdot)\right) .
\end{aligned}
$$

We call the problem above an LQ non-zero sum differential game with asymmetric information. For simplicity, we denote it by Problem (LQ NZSDG).

The LQ problems constitute an extremely important class of optimal control or differential game problems, since they can model many problems in applications, and also reasonably approximate nonlinear control or game problems. On the other hand, there also exist so called partial and asymmetric information problems in real world. For example, investors only partially know the information from security market (see [1,2]); in many situations, "insider trading" maybe exist, which means that the insider has access to material and non-public information about the security and the available information is asymmetric between the insider and the common trader (see, e.g., $[3,4]$ ); the principal faces information asymmetric and risk with regards to whether the agent has effectively completed a contract, when a principal hires an agent to perform specific duties (see, e.g., $[5,6])$. For more information about LQ control or game problems, the interested readers may refer the following partial list of the works including [7-13] with complete information, and [14] with partial information, and the references therein.

It is very important and meaningful to find explicit Nash equilibrium points for differential game problems. When the available information is partial or asymmetric, we need to derive the corresponding optimal filtering of the states and adjoint variables which will be used to represent the Nash equilibrium points. It is very difficult to obtain the equations satisfied by the optimal filtering when the available information is asymmetric for Player 1 and Player 2. Up till now, it seems that there has been no literature about LQ differential games with asymmetric information $\mathscr{G}_{t}^{1}$ and $\mathscr{G}_{t}^{2}$. However, in case where $\mathscr{G}_{t}^{i}(i=1,2)$ are chosen as certain special forms, we can still derive the filtering equations and then obtain the explicit form of the Nash equilibrium point. In the sequel, we will study Problem (LQ NZSDG) under the following four classes of asymmetric information:

(i) $\mathscr{G}_{t}^{1}=\mathscr{F}_{t}^{1,2}$ and $\mathscr{G}_{t}^{2}=\mathscr{F}_{t}^{2,3}$; that is, the two players possess the common partial information $\mathscr{F}_{t}^{2}$;

(ii) $\mathscr{G}_{t}^{1}=\mathscr{F}_{t}^{1,2}$ and $\mathscr{G}_{t}^{2}=\mathscr{F}_{t}^{2}$; that is, Player 1 possesses more information than Player 2;

(iii) $\mathscr{G}_{t}^{1}=\mathscr{F}_{t}$ and $\mathscr{G}_{t}^{2}=\mathscr{F}_{t}^{2}$; that is, Player 1 possesses the full information and Player 2 possesses the partial informaion;

(iv) $\mathscr{G}_{t}^{1}=\mathscr{F}_{t}^{1,2}$ and $\mathscr{G}_{t}^{2}=\mathscr{F}_{t}^{3}$; that is, the two players possess the mutually independent information.

In Section 3, we will point out that some other cases similar to (i)-(iv) can be also solved by the same idea and method. To our knowledge, this paper is the first try to study LQ nonzero sum differential games in the setting of the asymmetric information.

The rest of this paper is organized as follows. In Section 2, we introduce some preliminaries which will be used to derive the forward-backward filtering equations and prove the corresponding existence and uniqueness of the solutions. In Section 3, we obtain the unique explicit Nash equilibrium point for each class of asymmetric information above. We also introduce some Riccati equations and represent the unique Nash equilibrium point in a feedback form of the optimal filtering of the state with respect to the corresponding 
asymmetric information, through the solutions of the Riccati equations. Some conclusions are given in Section 4.

\section{Preliminary Results}

In this section, we are going to introduce two lemmas, which will be often used later. First, we present existence and uniqueness for the solutions of the forward-backward stochastic differential equation (FBSDE, for short), whose dynamics is described by

$$
\begin{gathered}
d x=b(t, x, y) d t+\sigma(t, x, y) d W \\
-d y=f(t, x, y, z) d t-z d W, \\
x(0)=x_{0}, \quad y(T)=\varphi(x(T)) .
\end{gathered}
$$

Here $x(\cdot)$ satisfies an (forward) SDE, $(y(\cdot), z(\cdot))$ satisfies a backward stochastic differential equation, $W(\cdot)$ is a $d$ dimensional standard Brownian motion, $(x, y, z)$ takes value in $R^{n} \times R^{n} \times R^{n \times d}$, and $b, \sigma, f$, and $\varphi$ are the mappings with suitable sizes.

We introduce the notations

$$
u=(x, y, z)^{*}, \quad A(t, u)=(-f, b, \sigma)^{*}(t, u),
$$

and make the following assumption.

$\left(\mathrm{H}_{1}\right) A(t, u)$ and $\varphi$ are uniformly Lipschitz continuous with respect to their variables; for each $x, \varphi(x)$ is in $\mathscr{L}_{\mathscr{F}_{T}}^{2}\left(\Omega ; R^{n}\right)$; for every $(\omega, t) \in \Omega \times$ $[0, T], b(\omega, t, 0,0) \in \mathscr{L}_{\mathscr{F}}^{2}\left(0, T ; R^{n}\right), \sigma(\omega, t, 0,0) \quad \epsilon$ $\mathscr{L}_{\mathscr{F}}^{2}\left(0, T ; R^{n \times d}\right)$, and $f(\omega, t, 0,0,0) \in \mathscr{L}_{\mathscr{F}}^{2}\left(0, T ; R^{n}\right)$.

We also make the following assumption.

$\left(\mathrm{H}_{2}\right)$ The functions $A(t, u)$ and $\varphi$ satisfy the monotonic conditions:

$$
\begin{gathered}
\left\langle A\left(t, u_{1}\right)-A\left(t, u_{2}\right), u_{1}-u_{2}\right\rangle \\
\leq-\kappa_{1}\left|x_{1}-x_{2}\right|^{2}-\kappa_{2}\left|y_{1}-y_{2}\right|^{2}, \\
\left\langle\varphi\left(x_{1}\right)-\varphi\left(x_{2}\right), x_{1}-x_{2}\right\rangle \geq \kappa_{3}\left|x_{1}-x_{2}\right|^{2}, \\
\forall u_{1}-u_{2}=\left(x_{1}-x_{2}, y_{1}-y_{2}, z_{1}-z_{2}\right),
\end{gathered}
$$

where $\kappa_{1}, \kappa_{1}$, and $\kappa_{3}$ are given nonnegative constants satisfying $\kappa_{1}+\kappa_{2}>0, \kappa_{2}+\kappa_{3}>0$.

Then we have the following lemma, which is a direct deduction of Theorem 1 in $\mathrm{Wu}$ and $\mathrm{Yu}$ [11] with no random jumps.

Lemma 1. If the assumptions $\left(H_{1}\right)$ and $\left(H_{2}\right)$ hold, then (6) has a unique triple $(x(\cdot), y(\cdot), z(\cdot)) \in \mathscr{L}_{\mathscr{F}_{t}}^{2}\left(0, T ; R^{n+n+n \times d}\right)$.
Remark 2. If we assume $\sigma \equiv 0$ and all functions are deterministic, then (6) is reduced to a forward-backward ordinary differential equation (ODE, for short):

$$
\begin{gathered}
d x=b(t, x, y) d t, \\
-d y=f(t, x, y) d t, \\
x(0)=x_{0}, \quad y(T)=\varphi(x(T)) .
\end{gathered}
$$

We define the notation $u=(x, y)^{*}, G(t, u)=(-f, b)^{*}(t, u)$. If $b, f, \varphi$, and $G$ satisfy the assumptions $\left(\mathrm{H}_{1}\right)$ and $\left(\mathrm{H}_{2}\right)$ with $\mathscr{L}_{\mathscr{F}_{t}}^{2}(0, T ; S)$ replaced by $\mathscr{L}^{2}(0, T ; S)$ and $\varphi$ is uniformly bounded, then (9) has a unique solution $(x, y) \in$ $\mathscr{L}^{2}\left(0, T ; \mathbf{R}^{n+n}\right)$.

The following lemma is from the monograph by Chung [15] (see the example, Section 9.2).

Lemma 3. If $\mathscr{F}_{1}, \mathscr{F}_{2}$, and $\mathscr{F}_{3}$ are three $\sigma$-algebras, and $\mathscr{F}_{1} \mathrm{~V}$ $\mathscr{F}_{2}$ is independent of $\mathscr{F}_{3}$, then, for any integrable random variable $X \in \mathscr{F}_{1}$, we have $\mathbb{E}\left[X \mid \mathscr{F}_{2} \vee \mathscr{F}_{3}\right]=\mathbb{E}\left[X \mid \mathscr{F}_{2}\right]$.

\section{Nash Equilibrium Point}

In this section, we will derive the explicit form of the Nash equilibrium point for Problem (LQ NZSDG), applying stochastic maximum principle for partial information optimal control problem and the technique of complete square. Further, we also introduce the Riccati equations and represent the Nash equilibrium point as a feedback of the optimal filters $\widehat{x}, \tilde{x}$, and $\bar{x}$, through the solutions to the Riccati equations.

We first introduce two LQ stochastic control problems with two pieces of general asymmetric information $\mathscr{G}_{t}^{1}$ and $\mathscr{G}_{t}^{2}$ which is closely related to problem (LQ NZSDG).

Problem (LQSC1):

$$
\begin{gathered}
\min \left\{J_{1}^{u_{2}}\left(v_{1}(\cdot)\right) \mid v_{1}(\cdot) \in \mathcal{U}_{1}\right\}, \\
J_{1}^{u_{2}}\left(v_{1}(\cdot)\right)=\frac{1}{2} \mathbb{E}\left[\int_{0}^{T}\left(l_{1}\left(x^{v_{1}}\right)^{2}+m_{1}\left(v_{1}\right)^{2}\right) d t+r_{1} x^{v_{1}}(T)^{2}\right],
\end{gathered}
$$

subject to

$$
\begin{aligned}
d x^{v_{1}}= & {\left[a x^{v_{1}}+b_{1} v_{1}(t)+b_{2} u_{2}+c\right] d t } \\
& +g_{1} d W_{1}+\left[e x^{v_{1}}+g_{2}\right] d W_{2}+g_{3} d W_{3}, \\
x^{v_{1}}(0)= & x_{0} .
\end{aligned}
$$

Problem (LQSC2):

$$
\min \left\{J_{2}^{u_{1}}\left(v_{2}(\cdot)\right) \mid v_{2}(\cdot) \in \mathcal{U}_{2}\right\},
$$

$$
J_{2}^{u_{1}}\left(v_{2}(\cdot)\right)=\frac{1}{2} \mathbb{E}\left[\int_{0}^{T}\left(l_{2}\left(x^{v_{2}}\right)^{2}+m_{2}\left(v_{2}\right)^{2}\right) d t+r_{2} x^{v_{2}}(T)^{2}\right],
$$


subject to

$$
\begin{aligned}
d x^{v_{2}}= & {\left[a x^{v_{2}}+b_{1} u_{1}(t)+b_{2} v_{2}+c\right] d t } \\
& +g_{1} d W_{1}+\left[e x^{v_{2}}+g_{2}\right] d W_{2}+g_{3} d W_{3}, \\
x^{v_{2}}(0)= & x_{0} .
\end{aligned}
$$

We can check that $x^{u_{1}}=x^{u_{2}}=x^{u_{1}, u_{2}} \equiv x, J_{1}^{u_{2}}\left(u_{1}(\cdot)\right)=$ $J_{1}\left(u_{1}(\cdot), u_{2}(\cdot)\right)$, and $J_{2}^{u_{1}}\left(u_{2}(\cdot)\right)=J_{2}\left(u_{1}(\cdot), u_{2}(\cdot)\right)$ hold. If $\left(u_{1}, u_{2}\right)$ is a Nash equilibrium point, then, from the definition of Nash equilibrium point (see (5)), we can conclude that $u_{1}$ (resp., $u_{2}$ ) is an optimal control for Problem (LQSC1) (resp., Problem (LQSC2)). Appealing to the stochastic maximum principle under partial information (see [16], Remark 2.1 with the drift coefficient of the observation equation being zero and convex control domain, or [17], Theorem 3.1 with nonrandom jumps), we can derive the following necessary conditions of the optimal controls for Problem (LQSC1) and Problem (LQSC2).

Lemma 4. If $u_{1}$ (resp., $u_{2}$ ) is an optimal control for Problem (LQSC1) (resp., Problem (LQSC2)), then we have

$$
\begin{aligned}
& u_{1}(t)=-m_{1}^{-1}(t) b_{1}(t) \mathbb{E}\left(y_{1}(t) \mid \mathscr{G}_{t}^{1}\right), \\
& u_{2}(t)=-m_{2}^{-1}(t) b_{2}(t) \mathbb{E}\left(y_{2}(t) \mid \mathscr{G}_{t}^{2}\right),
\end{aligned}
$$

where $\left(x,\left(y_{1}, z_{11}, z_{12}, z_{13}\right),\left(y_{2}, z_{21}, z_{22}, z_{23}\right)\right)$ is a solution to the following FBSDE:

$$
\begin{gathered}
d x=\left[a x-b_{1}^{2} m_{1}^{-1} \mathbb{E}\left(y_{1}(t) \mid \mathscr{G}_{t}^{1}\right)-b_{2}^{2} m_{2}^{-1} \mathbb{E}\left(y_{2}(t) \mid \mathscr{G}_{t}^{2}\right)\right. \\
+c] d t+g_{1} d W_{1}+\left[e x+g_{2}\right] d W_{2}+g_{3} d W_{3}, \\
-d y_{1}=\left[a y_{1}+e z_{12}+l_{1} x\right] d t-z_{11} d W_{1}-z_{12} d W_{2}-z_{13} d W_{3}, \\
-d y_{2}=\left[a y_{2}+e z_{22}+l_{2} x\right] d t-z_{21} d W_{1}-z_{22} d W_{2}-z_{23} d W_{3}, \\
x(0)=x_{0}, \quad y_{1}(T)=r_{1} x(T), \quad y_{2}(T)=r_{2} x(T) .
\end{gathered}
$$

It is obvious that $\left(u_{1}, u_{2}\right) \in \mathscr{U}_{1} \times \mathscr{U}_{2}$ is a candidate Nash equilibrium point for Problem (LQ NZSDG). We will prove $\left(u_{1}, u_{2}\right)$ is exactly a Nash equilibrium point in the sequel.

Lemma 5. $\left(u_{1}, u_{2}\right)$ in (21) is indeed a Nash equilibrium point for Problem (LQ NZSDG).

Proof. For any $v_{1}(\cdot) \in \mathscr{U}_{1}$, we have

$$
\begin{aligned}
J_{1}( & \left.v_{1}(\cdot), u_{2}(\cdot)\right)-J_{1}\left(u_{1}(\cdot), u_{2}(\cdot)\right) \\
= & \frac{1}{2} \mathbb{E} \int_{0}^{T}\left[l_{1}\left(x^{v_{1}, u_{2}}-x\right)^{2}+m_{1}\left(v_{1}-u_{1}\right)^{2}\right] d t \\
& +\frac{1}{2} \mathbb{E}\left[r_{1}\left(x^{v_{1}, u_{2}}(T)-x(T)\right)^{2}\right]+\Theta,
\end{aligned}
$$

where

$$
\begin{aligned}
\Theta= & \mathbb{E} \int_{0}^{T}\left[l_{1} x\left(x^{v_{1}, u_{2}}-x\right)+m_{1} u_{1}\left(v_{1}-u_{1}\right)\right] d t \\
& +\mathbb{E}\left[r_{1} x(T)\left(x^{v_{1}, u_{2}}(T)-x(T)\right)\right] .
\end{aligned}
$$

We apply Itô's formula to $y_{1}\left(x^{v_{1}, u_{2}}-x\right)$ and get

$$
\begin{gathered}
\Theta=\mathbb{E} \int_{0}^{T}\left(m_{1}(t) u_{1}(t)+b_{1}(t) y_{1}(t)\right)\left(v_{1}(t)-u_{1}(t)\right) d t \\
=\mathbb{E} \int_{0}^{T} \mathbb{E}\left[\left(m_{1}(t) u_{1}(t)+b_{1}(t) y_{1}(t)\right)\right. \\
\left.\quad \times\left(v_{1}(t)-u_{1}(t)\right) \mid \mathscr{G}_{t}^{1}\right] d t \\
=\mathbb{E} \int_{0}^{T}\left(m_{1}(t) u_{1}(t)+b_{1}(t) \mathbb{E}\left(y_{1}(t) \mid \mathscr{G}_{t}^{1}\right)\right) \\
\quad \times\left(v_{1}(t)-u_{1}(t)\right) d t=0 .
\end{gathered}
$$

Then, because $l_{1}$ and $r_{1}$ are nonnegative, and $m_{1}$ is positive, we have

$$
J_{1}\left(v_{1}(\cdot), u_{2}(\cdot)\right)-J_{1}\left(u_{1}(\cdot), u_{2}(\cdot)\right) \geq 0 .
$$

Similarly, for any $v_{2}(\cdot) \in \mathscr{U}_{2}$, we also have

$$
J_{2}\left(u_{1}(\cdot), v_{2}(\cdot)\right)-J_{2}\left(u_{1}(\cdot), u_{2}(\cdot)\right) \geq 0 .
$$

Therefore, we can conclude that $\left(u_{1}, u_{2}\right)$ in (14) is a Nash equilibrium point for Problem (LQ NZSDG) indeed.

Combining Lemmas 4 and 5, we obtain the following theorem.

Theorem 6. $\left(u_{1}, u_{2}\right)$ is a Nash equilibrium point for Problem (LQ NZSDG) if and only if $\left(u_{1}, u_{2}\right)$ has the form denoted by (14) and $\left(x,\left(y_{1}, z_{11}, z_{12}, z_{13}\right),\left(y_{2}, z_{21}, z_{22}, z_{23}\right)\right)$ satisfies FBSDE (15a)-(15d).

Remark 7. If (15a)-(15d) has a unique solution, then Problem (LQ NZSDG) has a unique Nash equilibrium point. If (15a)(15d) have many solutions, then Problem (LQ NZSDG) may have many Nash equilibrium points. If (22a)-(22d) have no solution, Problem (LQ NZSDG) has no Nash equilibrium point. The existence and uniqueness of the Nash equilibrium point for Problem ( $L Q N Z S D G$ ) are equivalent to the existence and uniqueness of (15a)-(15d).

Note that, under the two pieces of general asymmetric information $\mathscr{G}_{t}^{1}$ and $\mathscr{G}_{t}^{2}$, the optimal filtering $\mathbb{E}\left(y_{i}(t)\right.$ $\left.\mathscr{G}_{t}^{i}\right)(i=1,2)$ is very abstract which leads to the difficulty in finding the filtering equations satisfied by $\mathbb{E}\left(y_{i}(t)\right.$ । $\left.\mathscr{G}_{t}^{i}\right)(i=1,2)$. In the following, we begin to study Problem (LQ NZSDG) under several classes of particular asymmetric information. Though the chosen observable information is a bit special, the mathematical deductions are still highly complicated, and the derived results are interesting and meaningful. 
3.1. Case 1: $\mathscr{G}_{t}^{1}=\mathscr{F}_{t}^{1,2}$ and $\mathscr{G}_{t}^{2}=\mathscr{F}_{t}^{2,3}$. In this case, from the notations defined by (1), we have $\mathbb{E}\left(y_{1}(t) \mid \mathscr{G}_{t}^{1}\right)=\widehat{y}_{1}(t)$ and $\mathbb{E}\left(y_{2}(t) \mid \mathscr{G}_{t}^{2}\right)=\bar{y}_{2}(t)$. Hereinafter, we simply call $\hat{y}_{1}$ and $\bar{y}_{2}$ the optimal filters of $y_{1}$ and $y_{2}$, respectively, if there is no ambiguity from the notations and context. Then Theorem 6 can be rewritten as follows.

Theorem 8. $\left(u_{1}, u_{2}\right)$ is a Nash equilibrium point for Problem (LQ NZSDG) if and only if $\left(u_{1}, u_{2}\right)$ has the following form:

$$
\begin{aligned}
& u_{1}(t)=-m_{1}^{-1}(t) b_{1}(t) \hat{y}_{1}(t), \\
& u_{2}(t)=-m_{2}^{-1}(t) b_{2}(t) \bar{y}_{2}(t),
\end{aligned}
$$

where $\left(x,\left(y_{1}, z_{11}, z_{12}, z_{13}\right),\left(y_{2}, z_{21}, z_{22}, z_{23}\right)\right)$ is a solution to the following FBSDE:

$$
\begin{gathered}
d x=\left[a x-b_{1}^{2} m_{1}^{-1} \hat{y}_{1}-b_{2}^{2} m_{2}^{-1} \bar{y}_{2}+c\right] d t \\
+g_{1} d W_{1}+\left[e x+g_{2}\right] d W_{2}+g_{3} d W_{3}, \\
-d y_{1}=\left[a y_{1}+e z_{12}+l_{1} x\right] d t-z_{11} d W_{1}-z_{12} d W_{2}-z_{13} d W_{3}, \\
-d y_{2}=\left[a y_{2}+e z_{22}+l_{2} x\right] d t-z_{21} d W_{1}-z_{22} d W_{2}-z_{23} d W_{3}, \\
x(0)=x_{0}, \quad y_{1}(T)=r_{1} x(T), \quad y_{2}(T)=r_{2} x(T) .
\end{gathered}
$$

We can see that $(22 \mathrm{a})-(22 \mathrm{~d})$ is a very complicated FBSDE. First, (forward) SDE (22a) is one dimensional and the combination of BSDEs (22b) and (22c) is two dimensional, which is more intricate than the case of forward SDE and BSDE with the same dimension. Second, the drift terms and terminal conditions in (22b) and (22c) contain $x$. Finally, the drift term in (22a) contains the optimal filter $\widehat{y}_{1}$ (resp., $\bar{y}_{2}$ ) of $y_{1}$ (resp., $y_{2}$ ) with respect to $\mathscr{F}_{t}^{1,2}$ (resp., $\mathscr{F}_{t}^{2,3}$ ), whose dynamics has not been known.

Now it is the position to seek the dynamics of $\widehat{y}_{1}(t)$ and $\bar{y}_{2}(t)$ which will be used to construct the analytical representation of the Nash equilibrium point. Applying Lemma 5.4 in Xiong [18] and Lemma 3, we obtain the optimal filters of $x$ and $y_{1}$ in $(22 \mathrm{a})$ and $(22 \mathrm{~b})$ with respect to $\mathscr{F}_{t}^{1,2}$ for Player 1 which satisfies

$$
\begin{gathered}
d \hat{x}=\left[a \widehat{x}-b_{1}^{2} m_{1}^{-1} \widehat{y}_{1}-b_{2}^{2} m_{2}^{-1} \tilde{y}_{2}+c\right] d t \\
+g_{1} d W_{1}+\left[e \widehat{x}+g_{2}\right] d W_{2}, \\
-d \widehat{y}_{1}=\left[a \widehat{y}_{1}+e \widehat{z}_{12}+l_{1} \widehat{x}\right] d t-\widehat{z}_{11} d W_{1}-\widehat{z}_{12} d W_{2}, \\
\widehat{x}(0)=x_{0}, \quad \widehat{y}_{1}(T)=r_{1} \widehat{x}(T) .
\end{gathered}
$$

Similarly, we can obtain the optimal filters of $x$ and $y_{2}$ in (22a) and (22c) with respect to $\mathscr{F}_{t}^{2,3}$ for Player 2 which satisfies

$$
\begin{gathered}
d \bar{x}=\left[a \bar{x}-b_{1}^{2} m_{1}^{-1} \tilde{y}_{1}-b_{2}^{2} m_{2}^{-1} \bar{y}_{2}+c\right] d t \\
+\left[e \bar{x}+g_{2}\right] d W_{2}+g_{3} d W_{3}, \\
-d \bar{y}_{2}=\left[a \bar{y}_{2}+e \bar{z}_{22}+l_{2} \bar{x}\right] d t-\bar{z}_{22} d W_{2}-\bar{z}_{23} d W_{3}, \\
\bar{x}(0)=x_{0}, \quad \bar{y}_{2}(T)=r_{2} \bar{x}(T) .
\end{gathered}
$$

Note that (23a) and (24a) involve the optimal filter $\tilde{y}_{i}$ of $y_{i}$ with respect to $\mathscr{F}_{t}^{2}$; that is, $\widetilde{y}_{i}(t)=\mathbb{E}\left(y_{i}(t) \mid \mathscr{F}_{t}^{2}\right)(i=1,2)$. We can derive that $\tilde{y}_{2}$ and $\tilde{y}_{1}$ together with the optimal filter $\tilde{x}$ of $x$ satisfy

$$
\begin{gathered}
d \tilde{x}=\left[a \tilde{x}-b_{1}^{2} m_{1}^{-1} \tilde{y}_{1}-b_{2}^{2} m_{2}^{-1} \tilde{y}_{2}+c\right] d t+\left[e \tilde{x}+g_{2}\right] d W_{2}, \\
-d \tilde{y}_{1}=\left[a \tilde{y}_{1}+e \tilde{z}_{12}+l_{1} \tilde{x}\right] d t-\widetilde{z}_{12} d W_{2}, \\
-d \tilde{y}_{2}=\left[a \tilde{y}_{2}+e \tilde{z}_{22}+l_{2} \tilde{x}\right] d t-\tilde{z}_{22} d W_{2}, \\
\tilde{x}(0)=x_{0}, \quad \tilde{y}_{1}(T)=r_{1} \tilde{x}(T), \quad \tilde{y}_{2}(T)=r_{2} \tilde{x}(T) .
\end{gathered}
$$

Note that (23a)-(25d) are coupled forward-backward stochastic filtering equations. It is remarkable that the filtering equations are essentially different from the classical ones of SDEs, and the main reason is that BSDEs are included in the equations. To our best knowledge, this class of filtering equations is originally found by Huang et al. [19] when they studied the partial information control problems of backward stochastic systems. This class of filtering equations is later also discussed when some authors investigated the optimal control or differential games of partial informatio in BSDEs or FBSDEs (see [20-26]).

We introduce an assumption:

$$
\left(\mathrm{H}_{3}\right) b_{1}^{2}(t) m_{1}^{-1}(t)=b_{2}^{2}(t) m_{2}^{-1}(t), t \in[0, T]
$$

which is needed in the following lemmas and theorems.

Lemma 9. Under the assumption $\left(H_{3}\right)$, (25a)-(25d) have a unique solution $\left(\widetilde{x},\left(\widetilde{y}_{1}, \widetilde{z}_{12}\right),\left(\widetilde{y}_{2}, \widetilde{z}_{22}\right)\right) \in \mathscr{L}_{\mathscr{F}_{t}^{2}}^{2}\left(0, T ; R^{5}\right)$.

Proof. We first introduce another FBSDE:

$$
\begin{gathered}
d n=\left(a n-b_{1}^{2} m_{1}^{-1} p+c\right) d t+\left(e n+g_{2}\right) d W_{2}, \\
-d p=\left(a p+e q+\left(l_{1}+l_{2}\right) n\right) d t-q d W_{2}, \\
n(0)=x_{0}, \quad p(T)=\left(r_{1}+r_{2}\right) n(T) .
\end{gathered}
$$

If $\left(\tilde{x},\left(\tilde{y}_{1}, \widetilde{z}_{12}\right),\left(\tilde{y}_{2}, \widetilde{z}_{22}\right)\right)$ is a solution to $(25 \mathrm{a})-(25 \mathrm{~d})$, then $(n, p, q)$ is a solution to $(26)$, where

$$
n=\tilde{x}, \quad p=\tilde{y}_{1}+\tilde{y}_{2}, \quad q=\widetilde{z}_{12}+\widetilde{z}_{22} \text {. }
$$


On the other hand, if $(n, p, q)$ is a solution to (26), we introduce the following BSDE:

$$
\begin{aligned}
& -d p_{1}=\left[a p_{1}+e q_{12}+l_{1} n\right] d t-q_{12} d W_{2}, \\
& -d p_{2}=\left[a p_{2}+e q_{22}+l_{2} n\right] d t-q_{22} d W_{2}, \\
& p_{1}(T)=r_{1} n(T), \quad p_{2}(T)=r_{2} n(T) .
\end{aligned}
$$

From the existence and uniqueness of BSDE (see [27]), (28) has a unique solution $\left(p_{1}, q_{12}, p_{2}, q_{22}\right)$ with $p_{1}+p_{2}=p, q_{12}+$ $q_{22}=q$. Further, we can check that $\left(n,\left(p_{1}, q_{12}\right),\left(p_{2}, q_{22}\right)\right)$ is a solution to (25a)-(25d). In other words, the existence and uniqueness of (25a)-(25d) are equivalent to those of (26). It is easy to check that $(26)$ satisfies the assumptions $\left(\mathrm{H}_{1}\right)$ and $\left(\mathrm{H}_{2}\right)$. From Lemma 1 , it has a unique solution $(n, p, q)$. So do (25a)-(25d).

We observe that (25a)-(25d) are independent of (23a)(23c) and (24a)-(24c). We can first solve (25a)-(25d) and derive the unique solution $\left(\tilde{x},\left(\tilde{y}_{1}, \widetilde{z}_{12}\right),\left(\tilde{y}_{2}, \widetilde{z}_{22}\right)\right)$. Then we plug $\tilde{y}_{2}$ (resp., $\tilde{y}_{1}$ ) into (23a)-(23c) (resp., (24a)-(24c)). From Lemma 1 , we have the following lemma.

Lemma 10. If the assumption $\left(H_{3}\right)$ holds, there exists a unique solution $\left(\widehat{x}, \widehat{y}_{1}, \widehat{z}_{11}, \widehat{z}_{12}\right) \in \mathscr{L}_{\mathscr{F}_{t}^{1,2}}^{2}\left(0, T ; R^{4}\right)$ (resp., $\left.\left(\bar{x}, \bar{y}_{2}, \bar{z}_{22}, \bar{z}_{23}\right) \in \mathscr{L}_{\mathscr{F}_{t}^{2,3}}^{2}\left(0, T ; R^{4}\right)\right)$ to (23a)-(23c) (resp., (24a)(24c)).

After we obtain the unique solutions $\widehat{y}_{1}$ and $\bar{y}_{2}$ by solving (23a) $-(23 c)$ and (24a)-(24c), respectively, from the existence and uniqueness of solutions of SDEs, we conclude that (22a) has a unique solution $x$. Further, (22b) and (22c) also have unique solutions $\left(y_{1}, z_{11}, z_{12}, z_{13}\right)$ and $\left(y_{2}, z_{21}, z_{22}, z_{23}\right)$, respectively. Then we can say that $(22 \mathrm{a})-(22 \mathrm{~d})$ have a unique solution, which implies the following theorem.

Theorem 11. Under the assumption $\left(H_{3}\right)$, Problem (LQ NZSDG) has a unique Nash equilibrium point denoted by

$$
\begin{aligned}
& u_{1}(t)=-m_{1}^{-1}(t) b_{1}(t) \hat{y}_{1}(t), \\
& u_{2}(t)=-m_{2}^{-1}(t) b_{2}(t) \bar{y}_{2}(t),
\end{aligned}
$$

where $\hat{y}_{1}$ and $\bar{y}_{2}$ are uniquely determined by the systems of (23a)-(25d).

In the following, the Riccati equations are introduced, and the Nash equilibrium point is represented in a feedback of the optimal filters $\widehat{x}, \tilde{x}$, and $\bar{x}$. Hereinafter, we suppose the assumption $\left(\mathrm{H}_{3}\right)$ always holds.

Set

$$
\tilde{y}_{i}=\alpha_{i} \tilde{x}+\beta_{i} \quad(i=1,2)
$$

where $\alpha_{i}$ and $\beta_{i}$ are undetermined deterministic functions on $[0, T]$ satisfying $\alpha_{i}(T)=r_{i}$ and $\beta_{i}(T)=0$.
Applying Itô's formula to $\tilde{y}_{1}$ in $(30)$, it yields

$$
\begin{aligned}
d \tilde{y}_{1}= & {\left[\left(\dot{\alpha}_{1}-b_{1}^{2} m_{1}^{-1} \alpha_{1}^{2}-b_{2}^{2} m_{2}^{-1} \alpha_{1} \alpha_{2}+a \alpha_{1}\right) \tilde{x}\right.} \\
& \left.+\left(\dot{\beta}_{1}-b_{1}^{2} m_{1}^{-1} \alpha_{1} \beta_{1}-b_{2}^{2} m_{2}^{-1} \alpha_{1} \beta_{2}+\alpha_{1} c\right)\right] d t \\
& +\alpha_{1}\left(e \tilde{x}+g_{2}\right) d W_{2},
\end{aligned}
$$

which implies

$$
\widetilde{z}_{12}=\alpha_{1}\left(e \tilde{x}+g_{2}\right)
$$

Substituting (30) and (32) into (25b) and comparing the coefficients between (25b) and (31), we have

$$
\begin{aligned}
& \dot{\alpha}_{1}-b_{1}^{2} m_{1}^{-1} \alpha_{1}^{2}+\left(2 a+e^{2}\right) \alpha_{1}-b_{2}^{2} m_{2}^{-1} \alpha_{1} \alpha_{2}+l_{1}=0, \quad \\
& \dot{\beta}_{1}+\left(a-b_{1}^{2} m_{1}^{-1} \alpha_{1}\right) \beta_{1}-b_{2}^{2} m_{2}^{-1} \alpha_{1} \beta_{2}+\left(c+e g_{2}\right) \alpha_{1}=0 .
\end{aligned}
$$

Applying Itô's formula to $\tilde{y}_{2}$ in (30), it yields

$$
\begin{aligned}
d \tilde{y}_{2}= & {\left[\left(\dot{\alpha}_{2}-b_{2}^{2} m_{2}^{-1} \alpha_{2}^{2}-b_{1}^{2} m_{1}^{-1} \alpha_{1} \alpha_{2}+a \alpha_{2}\right) \tilde{x}\right.} \\
& \left.+\left(\dot{\beta}_{2}-b_{2}^{2} m_{2}^{-1} \alpha_{2} \beta_{2}-b_{1}^{2} m_{1}^{-1} \alpha_{2} \beta_{1}+\alpha_{2} c\right)\right] d t \\
& +\alpha_{2}\left(e \tilde{x}+g_{2}\right) d W_{2},
\end{aligned}
$$

which implies

$$
\widetilde{z}_{22}=\alpha_{2}\left(e \tilde{x}+g_{2}\right)
$$

Substituting (30) and (35) into (25c) and comparing the coefficients between (25c) and (34), we have

$$
\begin{aligned}
& \dot{\alpha}_{2}-b_{2}^{2} m_{2}^{-1} \alpha_{2}^{2}+\left(2 a+e^{2}\right) \alpha_{2}-b_{1}^{2} m_{1}^{-1} \alpha_{1} \alpha_{2}+l_{2}=0, \quad \text { (36a) } \\
& \dot{\beta}_{2}+\left(a-b_{2}^{2} m_{2}^{-1} \alpha_{2}\right) \beta_{2}-b_{1}^{2} m_{1}^{-1} \alpha_{2} \beta_{1}+\left(c+e g_{2}\right) \alpha_{2}=0 .
\end{aligned}
$$

Let $\alpha=\alpha_{1}+\alpha_{2}$. From $\left(\mathrm{H}_{3}\right)$, we have

$$
\begin{array}{r}
\dot{\alpha}-b_{1}^{2} m_{1}^{-1} \alpha^{2}+\left(2 a+e^{2}\right) \alpha+l_{1}+l_{2}=0 \\
\text { on }[0, T), \quad \alpha(T)=r_{1}+r_{2} .
\end{array}
$$

Since (37) is a standard Riccati equation, it has a unique solution $\alpha(\cdot)$. Introduce two auxiliary equations:

$$
\begin{array}{r}
\dot{\bar{\alpha}}_{1}+\left[\left(2 a+e^{2}\right)-b_{1}^{2} m_{1}^{-1} \alpha\right] \bar{\alpha}_{1}+l_{1}=0 \\
\text { on }[0, T), \quad \dot{\bar{\alpha}}_{1}(T)=r_{1}, \\
\dot{\bar{\alpha}}_{2}+\left[\left(2 a+e^{2}\right)-b_{2}^{2} m_{2}^{-1} \alpha\right] \bar{\alpha}_{2}+l_{2}=0 \\
\text { on }[0, T), \quad \dot{\bar{\alpha}}_{2}(T)=r_{2},
\end{array}
$$

where $\alpha$ is the solution to (37). Obviously, ODEs (38) and (39) have unique solutions $\bar{\alpha}_{1}$ and $\bar{\alpha}_{2}$, respectively. In addition, we can check that $\alpha_{1}$ and $\alpha_{2}$ in (33a) and (36a) are also 
the solutions to (38) and (39), respectively. From the uniqueness of solutions to (38) and (39), it follows that

$$
\bar{\alpha}_{1}=\alpha_{1}, \quad \bar{\alpha}_{2}=\alpha_{2},
$$

which implies in turn that (33a) and (36a) have the unique solutions to $\alpha_{1}$ and $\alpha_{2}$.

Let $\beta=\beta_{1}+\beta_{2}$, and then we have

$$
\begin{array}{r}
\dot{\beta}+\left(a-b_{1}^{2} m_{1}^{-1} \alpha\right) \beta+\left(c+e g_{2}\right) \alpha=0 \\
\text { on }[0, T), \quad \beta(T)=0,
\end{array}
$$

where $\alpha$ is the solution to (37). Note that ODE (41) has a unique solution $\beta$. Introduce two another auxiliary equations:

$$
\begin{array}{r}
\dot{\bar{\beta}}_{1}+a \bar{\beta}_{1}-b_{2}^{2} m_{2}^{-1} \alpha_{1} \beta+\left(c+e g_{2}\right) \alpha_{1}=0 \\
\text { on }[0, T), \quad \bar{\beta}_{1}(T)=0, \\
\dot{\bar{\beta}}_{2}+a \bar{\beta}_{2}-b_{1}^{2} m_{1}^{-1} \alpha_{2} \beta+\left(c+e g_{2}\right) \alpha_{2}=0 \\
\text { on }[0, T), \quad \bar{\beta}_{2}(T)=0,
\end{array}
$$

where $\alpha_{1}, \alpha_{2}$, and $\beta$ are the solutions to (38), (39), and (41), respectively. Similarly, we can prove that (33b) and (36b) also have unique solutions $\beta_{1}$ and $\beta_{2}$ satisfying

$$
\bar{\beta}_{1}=\beta_{1}, \quad \bar{\beta}_{2}=\beta_{2} \text {. }
$$

Based on the arguments above, we can derive the analytical expressions for $\alpha_{1}, \alpha_{2}, \beta_{1}, \beta_{2}, \alpha$, and $\beta$. Then (25a) can be rewritten as

$$
\begin{aligned}
d \tilde{x} & =\left[\left(a-b_{1}^{2} m_{1}^{-1} \alpha\right) \tilde{x}-b_{1}^{2} m_{1}^{-1} \beta+c\right] d t+\left[e \tilde{x}+g_{2}\right] d W_{2}, \\
\tilde{x}(0) & =x_{0}
\end{aligned}
$$

which has a unique solution

$$
\begin{aligned}
\tilde{x}(t)=\Gamma_{0}^{t} x_{0}+\int_{0}^{t} \Gamma_{s}^{t}[ & \left(c(s)-b_{1}^{2}(s) m_{1}^{-1}(s) \beta(s)\right. \\
& \left.\left.-e(s) g_{2}(s)\right) d s+g_{2}(s) d W_{2}(s)\right],
\end{aligned}
$$

with $\Gamma_{s}^{t}=\exp \left\{\int_{s}^{t}\left[a(r)-b_{1}^{2}(r) m_{1}^{-1}(r) \alpha(r)-(1 / 2) e^{2}(r)\right] d r+\right.$ $\left.\int_{s}^{t} e(r) d W_{2}(r)\right\}$.

From the uniqueness of $\alpha_{1}, \alpha_{2}, \beta_{1}, \beta_{2}$, and $\tilde{x}$, it follows that $\tilde{y}_{i}$ in (30) has a unique analytical expression.

Substituting $\tilde{y}_{2}$ in (30) into (23a)-(23c), we have

$$
\begin{gathered}
d \hat{x}=\left[a \widehat{x}-b_{1}^{2} m_{1}^{-1} \widehat{y}_{1}-b_{2}^{2} m_{2}^{-1} \alpha_{2} \tilde{x}-b_{2}^{2} m_{2}^{-1} \beta_{2}+c\right] d t \\
+g_{1} d W_{1}+\left[e \widehat{x}+g_{2}\right] d W_{2}, \\
-d \widehat{y}_{1}=\left[a \widehat{y}_{1}+e \widehat{z}_{12}+l_{1} \widehat{x}\right] d t-\widehat{z}_{11} d W_{1}-\widehat{z}_{12} d W_{2}, \\
\widehat{x}(0)=x_{0}, \quad \widehat{y}_{1}(T)=r_{1} \widehat{x}(T) .
\end{gathered}
$$

Set

$$
\widehat{y}_{1}=\gamma_{1} \widehat{x}+\gamma_{2} \tilde{x}+\gamma_{3}
$$

with $\gamma_{1}(T)=r_{1}, \gamma_{2}(T)=\gamma_{3}(T)=0$. Applying Itô's formula to $\widehat{y}_{1}$ in (47), we have

$$
\begin{aligned}
d \widehat{y}_{1}=[ & \left(\dot{\gamma}_{1}-b_{1}^{2} m_{1}^{-1} \gamma_{1}^{2}+a \gamma_{1}\right) \widehat{x} \\
& +\left(\dot{\gamma}_{2}+\left(a-b_{1}^{2} m_{1}^{-1} \alpha-b_{1}^{2} m_{1}^{-1} \gamma_{1}\right) \gamma_{2}\right. \\
& \left.\quad-b_{2}^{2} m_{2}^{-1} \alpha_{2} \gamma_{1}\right) \tilde{x} \\
& +\dot{\gamma}_{3}-b_{1}^{2} m_{1}^{-1} \gamma_{1} \gamma_{3}+\left(c-b_{2}^{2} m_{2}^{-1} \beta_{2}\right) \gamma_{1} \\
& \left.+\left(c-b_{1}^{2} m_{1}^{-1} \beta\right) \gamma_{2}\right] d t \\
+ & \gamma_{1} g_{1} d W_{1}+\left[\gamma_{1}\left(e \widehat{x}+g_{2}\right)+\gamma_{2}\left(e \tilde{x}+g_{2}\right)\right] d W_{2},
\end{aligned}
$$

which implies

$$
\widehat{z}_{11}=\gamma_{1} g_{1}, \quad \widehat{z}_{12}=\gamma_{1}\left(e \hat{x}+g_{2}\right)+\gamma_{2}\left(e \tilde{x}+g_{2}\right) .
$$

Substituting (47) and (49) into (46b) and comparing the drift and diffusion coefficients with (48), we conclude that

$$
\begin{gathered}
\dot{\gamma}_{1}-b_{1}^{2} m_{1}^{-1} \gamma_{1}^{2}+\left(2 a+e^{2}\right) \gamma_{1}+l_{1}=0 \\
\dot{\gamma}_{2}+\left(2 a+e^{2}-b_{1}^{2} m_{1}^{-1} \alpha-b_{1}^{2} m_{1}^{-1} \gamma_{1}\right) \gamma_{2}-b_{2}^{2} m_{2}^{-1} \alpha_{2} \gamma_{1}=0 \\
\dot{\gamma}_{3}+\left(a-b_{1}^{2} m_{1}^{-1} \gamma_{1}\right) \gamma_{3}+\left(c-b_{2}^{2} m_{2}^{-1} \beta_{2}+e g_{2}\right) \gamma_{1} \\
+\left(c-b_{1}^{2} m_{1}^{-1} \beta+e g_{2}\right) \gamma_{2}=0
\end{gathered}
$$

It is clear that there exists a unique solution $\left(\gamma_{1}, \gamma_{2}, \gamma_{3}\right)$ to (50a)-(50c). We denote

$$
f_{1} \triangleq-\left(b_{2}^{2} m_{2}^{-1} \alpha_{2}+b_{1}^{2} m_{1}^{-1} \gamma_{2}\right) \tilde{x}-b_{1}^{2} m_{1}^{-1} \gamma_{3}-b_{2}^{2} m_{2}^{-1} \beta_{2}+c,
$$

and then, in terms of (47), (46a) can be rewritten as

$$
d \widehat{x}=\left[\left(a-b_{1}^{2} m_{1}^{-1} \gamma_{1}\right) \hat{x}+f_{1}\right] d t+g_{1} d W_{1}+\left[e \widehat{x}+g_{2}\right] d W_{2},
$$
$\widehat{x}(0)=x_{0}$,

which has a unique solution:

$$
\begin{aligned}
\widehat{x}(t)=\Upsilon_{0}^{t} x_{0}+\int_{0}^{t} \Upsilon_{s}^{t}[ & \left(f_{1}(s)-e(s) g_{2}(s)\right) d s \\
& \left.\quad+g_{1}(s) d W_{1}(s)+g_{2}(s) d W_{2}(s)\right],
\end{aligned}
$$

with $\Upsilon_{s}^{t}=\exp \left\{\int_{s}^{t}\left[a(r)-b_{1}^{2}(r) m_{1}^{-1}(r) \gamma_{1}(r)-(1 / 2) e^{2}(r)\right] d r+\right.$ $\left.\int_{s}^{t} e(r) d W_{2}(r)\right\}$. 
Substituting $\widetilde{y}_{1}$ in (30) into (24a)-(24c), we have

$$
\begin{gathered}
d \bar{x}=\left[a \bar{x}-b_{1}^{2} m_{1}^{-1} \alpha_{1} \tilde{x}-b_{2}^{2} m_{2}^{-1} \bar{y}_{2}-b_{1}^{2} m_{1}^{-1} \beta_{1}+c\right] d t \\
+\left[e \bar{x}+g_{2}\right] d W_{2}+g_{3} d W_{3}, \\
-d \bar{y}_{2}=\left[a \bar{y}_{2}+e \bar{z}_{22}+l_{2} \bar{x}\right] d t-\bar{z}_{22} d W_{2}-\bar{z}_{23} d W_{3}, \\
\bar{x}(0)=x_{0}, \quad \bar{y}_{2}(T)=r_{2} \bar{x}(T) .
\end{gathered}
$$

Set

$$
\bar{y}_{2}=\tau_{1} \bar{x}+\tau_{2} \tilde{x}+\tau_{3},
$$

with $\tau_{1}(T)=r_{2}, \tau_{2}(T)=\tau_{3}(T)=0$. In the similar manner, we can deduce that $\left(\tau_{1}, \tau_{2}, \tau_{3}\right)$ satisfies

$$
\begin{gathered}
\dot{\tau}_{1}-b_{2}^{2} m_{2}^{-1} \tau_{1}^{2}+\left(2 a+e^{2}\right) \tau_{1}+l_{2}=0 \\
\dot{\tau}_{2}+\left(2 a+e^{2}-b_{1}^{2} m_{1}^{-1} \alpha-b_{2}^{2} m_{2}^{-1} \tau_{1}\right) \tau_{2}-b_{1}^{2} m_{1}^{-1} \alpha_{1} \tau_{1}=0 \\
\dot{\tau}_{3}+\left(a-b_{2}^{2} m_{2}^{-1} \tau_{1}\right) \tau_{3}+\left(c-b_{1}^{2} m_{1}^{-1} \beta_{1}+e g_{2}\right) \tau_{1} \\
+\left(c-b_{1}^{2} m_{1}^{-1} \beta+e g_{2}\right) \tau_{2}=0
\end{gathered}
$$

which has a unique solution $\left(\tau_{1}, \tau_{2}, \tau_{3}\right)$. We denote

$$
f_{2} \triangleq-\left(b_{2}^{2} m_{2}^{-1} \tau_{2}+b_{1}^{2} m_{1}^{-1} \alpha_{1}\right) \tilde{x}-b_{1}^{2} m_{1}^{-1} \beta_{1}-b_{2}^{2} m_{2}^{-1} \tau_{3}+c
$$

and then, in terms of (55), (54a) can be rewritten as

$$
\begin{aligned}
d \bar{x} & =\left[\left(a-b_{2}^{2} m_{2}^{-1} \tau_{1}\right) \bar{x}+f_{2}\right] d t+\left[e \bar{x}+g_{2}\right] d W_{2}+g_{3} d W_{3}, \\
\bar{x}(0) & =x_{0},
\end{aligned}
$$

which has a unique solution

$$
\begin{aligned}
\bar{x}(t)=\Psi_{0}^{t} x_{0}+\int_{0}^{t} \Psi_{s}^{t}[ & \left(f_{2}(s)-e(s) g_{2}(s)\right) d s \\
& \left.+g_{2}(s) d W_{2}(s)+g_{3}(s) d W_{3}(s)\right],
\end{aligned}
$$

with $\Psi_{s}^{t}=\exp \left\{\int_{s}^{t}\left[a(r)-b_{2}^{2}(r) m_{2}^{-1}(r) \tau_{1}(r)-(1 / 2) e^{2}(r)\right] d r+\right.$ $\left.\int_{s}^{t} e(r) d W_{2}(r)\right\}$.

Based on the arguments above, we derive the Nash equilibrium point which is represented in the feedback of the optimal filters $\widehat{x}, \tilde{x}$, and $\bar{x}$ of the state $x$. Then Theorem 11 can be rewritten as follows.

Theorem 12. Under the assumption $\left(\mathrm{H}_{3}\right)$, Problem (LQ NZSDG) has a unique Nash equilibrium point denoted by

$$
u_{1}(t)=-m_{1}^{-1}(t) b_{1}(t)\left(\gamma_{1}(t) \hat{x}(t)+\gamma_{2}(t) \tilde{x}(t)+\gamma_{3}(t)\right),
$$

$$
u_{2}(t)=-m_{2}^{-1}(t) b_{2}(t)\left(\tau_{1}(t) \bar{x}(t)+\tau_{2}(t) \tilde{x}(t)+\tau_{3}(t)\right) \text {, }
$$

where $\tilde{x}, \hat{x}$, and $\bar{x}$ are as shown in (45), (53), and (59), respectively, and $\gamma_{i}$ and $\tau_{i}(i=1,2,3)$ are uniquely determined by the systems of (50a)-(50c) and (56a)-(56c), respectively.

Remark 13. We introduce another assumption:

$$
\left(\mathrm{H}_{3}\right)^{\prime} b_{i}^{2} m_{i}^{-1}(i=1,2) \text { are independent of } t .
$$

We can check that when the assumption $\left(\mathrm{H}_{3}\right)$ is replaced by $\left(\mathrm{H}_{3}\right)^{\prime}$, the foregoing lemmas and theorems still hold.

3.2. Case 2: $\mathscr{G}_{t}^{1}=\mathscr{F}_{t}^{1,2}$ and $\mathscr{G}_{t}^{2}=\mathscr{F}_{t}^{2}$. In this case, we have $\mathbb{E}\left(y_{1}(t) \mid \mathscr{G}_{t}^{1}\right)=\widehat{y}_{1}(t)$ and $\mathbb{E}\left(y_{2}(t) \mid \mathscr{G}_{t}^{2}\right)=\tilde{y}_{2}(t)$. Applying the similar methods shown in Section 3.1, we can obtain the following theorem.

Theorem 14. $\left(u_{1}, u_{2}\right)$ is a Nash equilibrium point for Problem (LQ NZSDG) if and only if

$$
\begin{aligned}
& u_{1}(t)=-m_{1}^{-1}(t) b_{1}(t) \widehat{y}_{1}(t), \\
& u_{2}(t)=-m_{2}^{-1}(t) b_{2}(t) \tilde{y}_{2}(t),
\end{aligned}
$$

where $\left(x,\left(y_{1}, z_{11}, z_{12}, z_{13}\right),\left(y_{2}, z_{21}, z_{22}, z_{23}\right)\right)$ is a solution of the following FBSDE:

$$
\begin{gathered}
d x=\left[a x-b_{1}^{2} m_{1}^{-1} \widehat{y}_{1}-b_{2}^{2} m_{2}^{-1} \tilde{y}_{2}+c\right] d t \\
+g_{1} d W_{1}+\left[e x+g_{2}\right] d W_{2}+g_{3} d W_{3}, \\
-d y_{1}=\left[a y_{1}+e z_{12}+l_{1} x\right] d t \\
\quad-z_{11} d W_{1}-z_{12} d W_{2}-z_{13} d W_{3}, \\
-d y_{2}=\left[a y_{2}+e z_{22}+l_{2} x\right] d t \\
\quad-z_{21} d W_{1}-z_{22} d W_{2}-z_{23} d W_{3}, \\
x(0)=x_{0}, \quad y_{1}(T)=r_{1} x(T), \quad y_{2}(T)=r_{2} x(T) .
\end{gathered}
$$

Under the assumption $\left(\mathrm{H}_{3}\right)$, we can check that the filtering equations (23a)-(23c), (25a)-(25d), and the linear relations (30) and (47) still hold, and the systems of equations (33a), (33b), (36a), (36b), and (50a)-(50c) are still uniquely solvable. Then we have the following theorem.

Theorem 15. If $\left(\mathrm{H}_{3}\right)$ holds, then Problem (LQ NZSDG) has a unique Nash equilibrium point denoted by

$$
\begin{aligned}
& u_{1}(t)=-m_{1}^{-1}(t) b_{1}(t)\left(\gamma_{1}(t) \hat{x}(t)+\gamma_{2}(t) \tilde{x}(t)+\gamma_{3}(t)\right) \\
& u_{2}(t)=-m_{2}^{-1}(t) b_{2}(t)\left(\alpha_{2}(t) \tilde{x}(t)+\beta_{2}(t)\right)
\end{aligned}
$$

where $\tilde{x}$ and $\hat{x}$ are shown in (45) and (53), respectively. 
Remark 16. In the cases similar to Case 2 , such as $\mathscr{G}_{t}^{1}=\mathscr{F}_{t}^{2,3}$ and $\mathscr{G}_{t}^{2}=\mathscr{F}_{t}^{2}, \mathscr{G}_{t}^{1}=\mathscr{F}_{t}^{1,3}$, and $\mathscr{G}_{t}^{2}=\mathscr{F}_{t}{ }_{t}$, the corresponding results can be easily derived.

3.3. Case 3: $\mathscr{G}_{t}^{1}=\mathscr{F}_{t}$ and $\mathscr{G}_{t}^{2}=\mathscr{F}_{t}^{2}$. In this case, we have $\mathbb{E}\left(y_{1}(t) \mid \mathscr{G}_{t}^{1}\right)=y_{1}(t)$ and $\mathbb{E}\left(y_{2}(t) \mid \mathscr{G}_{t}^{2}\right)=\tilde{y}_{2}(t)$. Then we have the following theorem.

Theorem 17. $\left(u_{1}, u_{2}\right)$ is a Nash equilibrium point for Problem (LQ NZSDG) if and only if

$$
\begin{aligned}
& u_{1}(t)=-m_{1}^{-1}(t) b_{1}(t) y_{1}(t), \\
& u_{2}(t)=-m_{2}^{-1}(t) b_{2}(t) \tilde{y}_{2}(t),
\end{aligned}
$$

where $\left(x,\left(y_{1}, z_{11}, z_{12}, z_{13}\right),\left(y_{2}, z_{21}, z_{22}, z_{23}\right)\right)$ is a solution to the following FBSDE:

$$
\begin{aligned}
d x= & {\left[a x-b_{1}^{2} m_{1}^{-1} y_{1}-b_{2}^{2} m_{2}^{-1} \tilde{y}_{2}+c\right] d t+g_{1} d W_{1} } \\
& +\left[e x+g_{2}\right] d W_{2}+g_{3} d W_{3}, \\
-d y_{1}= & {\left[a y_{1}+e z_{12}+l_{1} x\right] d t-z_{11} d W_{1}-z_{12} d W_{2}-z_{13} d W_{3}, }
\end{aligned}
$$

Under the assumption $\left(H_{3}\right)$, we can check that $\left(\tilde{x},\left(\tilde{y}_{1}, \widetilde{z}_{12}\right),\left(\tilde{y}_{2}, \widetilde{z}_{22}\right)\right)$ still satisfies the filtering equations (25a)-(25d). From Section 3.1, we know that $\tilde{x}$ is shown as (45) and $\tilde{y}_{i}$ is uniquely represented by (30). Then (65a) can be rewritten as

$$
\begin{gathered}
d x=\left[a x-b_{1}^{2} m_{1}^{-1} y_{1}-b_{2}^{2} m_{2}^{-1} \alpha_{2} \tilde{x}-b_{2}^{2} m_{2}^{-1} \beta_{2}+c\right] d t \\
+g_{1} d W_{1}+\left[e x+g_{2}\right] d W_{2}+g_{3} d W_{3}, \\
-d y_{1}=\left[a y_{1}+e z_{12}+l_{1} x\right] d t-z_{11} d W_{1}-z_{12} d W_{2}-z_{13} d W_{3}, \\
x(0)=x_{0}, \quad y_{1}(T)=r_{1} x(T) .
\end{gathered}
$$

From Lemma 1, we can say that (66a)-(66c) has a unique solution $\left(x, y_{1}, z_{11}, z_{12}, z_{13}\right)$. Further, the relation between $y_{1}$ and $(x, \tilde{x})$ is as follows:

$$
y_{1}=\gamma_{1} x+\gamma_{2} \tilde{x}+\gamma_{3},
$$

where $\gamma_{i}(i=1,2,3)$ is the solution to (50a)-(50c), and

$$
\begin{array}{r}
x(t)=\Upsilon_{0}^{t} x_{0}+\int_{0}^{t} \Upsilon_{s}^{t}\left[\left(f_{1}(s)-e(s) g_{2}(s)\right) d s\right. \\
\left.+\sum_{i=1}^{3} g_{i}(s) d W_{i}(s)\right],
\end{array}
$$

with $\Upsilon_{s}^{t}=\exp \left\{\int_{s}^{t}\left[a(r)-b_{1}^{2}(r) m_{1}^{-1}(r) \gamma_{1}(r)-(1 / 2) e^{2}(r)\right] d r+\right.$ $\left.\int_{s}^{t} e(r) d W_{2}(r)\right\}$ and $f_{1}$ defined by (51). Then we have the following theorem.

Theorem 18. Under the assumptions $\left(\mathrm{H}_{3}\right)$, Problem (LQ NZSDG) has a unique Nash equilibrium point denoted by

$$
\begin{aligned}
& u_{1}(t)=-m_{1}^{-1}(t) b_{1}(t)\left(\gamma_{1}(t) x(t)+\gamma_{2}(t) \tilde{x}(t)+\gamma_{3}(t)\right), \\
& u_{2}(t)=-m_{2}^{-1}(t) b_{2}(t)\left(\alpha_{2}(t) \tilde{x}(t)+\beta_{2}(t)\right)
\end{aligned}
$$

where $\tilde{x}$ and $x$ are shown as (45) and (68), respectively.

Remark 19. In the cases similar to Case 3 , such as $\mathscr{G}_{t}^{1}=\mathscr{F}_{t}$ and $\mathscr{G}_{t}^{2}=\mathscr{F}_{t}^{1}, \mathscr{G}_{t}^{1}=\mathscr{F}_{t}$ and $\mathscr{G}_{t}^{2}=\mathscr{F}_{t}^{3}$, the corresponding results can be easily derived.

3.4. Case 4: $\mathscr{G}_{t}^{1}=\mathscr{F}_{t}^{1,2}$ and $\mathscr{G}_{t}^{2}=\mathscr{F}_{t}^{3}$. In this case, we have $\mathbb{E}\left(y_{1}(t) \mid \mathscr{G}_{t}^{1}\right)=\widehat{y}_{1}(t)$ and $\mathbb{E}\left(y_{2}(t) \mid \mathscr{G}_{t}^{2}\right)=\check{y}_{2}(t)$. Throughout this subsection, we make an additional assumption on (2):

$$
\left(\mathrm{H}_{4}\right) e(t)=0, t \in[0, T] \text {. }
$$

Similar to Sections 3.2 and 3.3, we directly present the following theorem.

Theorem 20. $\left(u_{1}, u_{2}\right)$ is a Nash equilibrium point for Problem (LQ NZSDG) if and only if

$$
\begin{aligned}
& u_{1}(t)=-m_{1}^{-1}(t) b_{1}(t) \widehat{y}_{1}(t), \\
& u_{2}(t)=-m_{2}^{-1}(t) b_{2}(t) \check{y}_{2}(t),
\end{aligned}
$$

where $\left(x,\left(y_{1}, z_{11}, z_{12}, z_{13}\right),\left(y_{2}, z_{21}, z_{22}, z_{23}\right)\right)$ is a solution to the following FBSDE:

$$
\begin{gathered}
d x=\left[a x-b_{1}^{2} m_{1}^{-1} \widehat{y}_{1}-b_{2}^{2} m_{2}^{-1} \check{y}_{2}+c\right] d t \\
+g_{1} d W_{1}+g_{2} d W_{2}+g_{3} d W_{3}, \\
-d y_{1}=\left[a y_{1}+l_{1} x\right] d t-z_{11} d W_{1}-z_{12} d W_{2}-z_{13} d W_{3}, \quad \\
-d y_{2}=\left[a y_{2}+l_{2} x\right] d t-z_{21} d W_{1}-z_{22} d W_{2}-z_{23} d W_{3}, \quad \\
x(0)=x_{0}, \quad y_{1}(T)=r_{1} x(T), \quad y_{2}(T)=r_{2} x(T) .
\end{gathered}
$$

Using the similar method shown in Section 3.1, we obtain the optimal filters of $x$ and $y_{1}$ in (71a) and (71b) with respect to $\mathscr{F}_{t}^{1,2}$ which satisfies

$$
\begin{array}{r}
d \widehat{x}=\left[a \widehat{x}-b_{1}^{2} m_{1}^{-1} \widehat{y}_{1}-b_{2}^{2} m_{2}^{-1} \mathbb{E} y_{2}+c\right] d t+g_{1} d W_{1}+g_{2} d W_{2}, \\
-d \widehat{y}_{1}=\left[a \widehat{y}_{1}+l_{1} \widehat{x}\right] d t-\widehat{z}_{11} d W_{1}-\widehat{z}_{12} d W_{2}, \\
\widehat{x}(0)=x_{0}, \quad \widehat{y}_{1}(T)=r_{1} \widehat{x}(T) .
\end{array}
$$

Here we denote by $\mathbb{E} \eta$ the mathematical expectation $\mathbb{E}(\eta(t))$ of the variable $\eta(t)$ and omit $t$ for simplicity. Similarly, we can 
obtain the optimal filters of $x$ and $y_{2}$ in (71a) and (71c) with respect to $\mathscr{F}_{t}^{3}$ which satisfy

$$
\begin{gathered}
d \check{x}=\left[a \check{x}-b_{1}^{2} m_{1}^{-1} \mathbb{E} y_{1}-b_{2}^{2} m_{2}^{-1} \check{y}_{2}+c\right] d t+g_{3} d W_{3}, \\
-d \check{y}_{2}=\left[a \check{y}_{2}+l_{2} \check{x}\right] d t-\check{z}_{23} d W_{3}, \\
\check{x}(0)=x_{0}, \quad \check{y}_{2}(T)=r_{2} \check{x}(T) .
\end{gathered}
$$

In addition, $\mathbb{E} y_{1}$ and $\mathbb{E} y_{2}$ together with $\mathbb{E} x$ satisfy

$$
\begin{gathered}
\dot{\mathbb{E}} x=a \mathbb{E} x-b_{1}^{2} m_{1}^{-1} \mathbb{E} y_{1}-b_{2}^{2} m_{2}^{-1} \mathbb{E} y_{2}+c, \\
-\dot{\mathbb{E}} y_{1}=a \mathbb{E} y_{1}+l_{1} \mathbb{E} x, \\
-\dot{\mathbb{E}} y_{2}=a \mathbb{E} y_{2}+l_{2} \mathbb{E} x, \\
\mathbb{E} x(0)=x_{0}, \quad \mathbb{E} y_{1}(T)=r_{1} \mathbb{E} x(T), \\
\mathbb{E} y_{2}(T)=r_{2} \mathbb{E} x(T),
\end{gathered}
$$

where $\dot{\mathbb{E}} \eta$ denotes $d \mathbb{E}(\eta(t)) / d t$ for $\eta=x, y_{1}, y_{2}$.

It is clear that (74a)-(74d) are a forward-backward ODE independent of (72a)-(73c). Using the similar method shown in Lemma 9 and Remark 2, we conclude that (74a)- (74d) have a unique solution $\left(\mathbb{E} x, \mathbb{E} y_{1}\right.$, and $\left.\mathbb{E} y_{2}\right)$. Plugging the solutions $\mathbb{E} y_{2}$ and $\mathbb{E} y_{1}$ into $(72 \mathrm{a})-(72 \mathrm{c})$ and $(73 \mathrm{a})-(73 \mathrm{c})$, respectively, and applying Lemma 1 , we conclude that $(72 \mathrm{a})-(73 \mathrm{c})$ have the unique solutions $\left(\widehat{x}, \widehat{y}_{1}, \widehat{z}_{11}, \widehat{z}_{12}\right)$ and $\left(\check{x}, \check{y}_{2}, \check{z}_{23}\right)$, respectively. Then we derive the more explicit representation of the Nash equilibrium point in (70) as follows.

Theorem 21. Under the assumptions $\left(\mathrm{H}_{3}\right)$ and $\left(\mathrm{H}_{4}\right)$, Problem (LQ NZSDG) has a unique Nash equilibrium point denoted by

$$
\begin{aligned}
& u_{1}(t)=-m_{1}^{-1}(t) b_{1}(t) \hat{y}_{1}(t), \\
& u_{2}(t)=-m_{2}^{-1}(t) b_{2}(t) \check{y}_{2}(t),
\end{aligned}
$$

where $\widehat{y}_{1}$ and $\check{y}_{2}$ are uniquely determined by the systems of (72a)-(74d).

In the sequel, we only present the results and omit the deduction procedures, because the method and technique are parallel to those in Section 3.1.

The relation between $\mathbb{E} y_{i}$ and $\mathbb{E} x$ is as follows:

$$
\mathbb{E} y_{i}=\alpha_{i} \mathbb{E} x+\beta_{i} \quad(i=1,2),
$$

where $\alpha_{i}, \beta_{i}, \alpha$, and $\beta$ are the unique solutions to the systems of (33a), (33b), (36a), (36b), (37), and (41) with $e(\cdot)$ replaced by 0 , respectively, and

$$
\mathbb{E} x(t)=\Gamma_{0}^{t} x_{0}+\int_{0}^{t} \Gamma_{s}^{t}\left[\left(c(s)-b_{1}^{2}(s) m_{1}^{-1}(s) \beta(s)\right)\right] d s
$$

with $\Gamma_{s}^{t}=\exp \left\{\int_{s}^{t}\left[a(r)-b_{1}^{2}(r) m_{1}^{-1}(r) \alpha(r)\right] d r\right\}$.

The relation between $\widehat{y}_{1}$ and $(\widehat{x}, \mathbb{E} x)$ is as follows:

$$
\widehat{y}_{1}=\gamma_{1} \hat{x}+\gamma_{2} \mathbb{E} x+\gamma_{3},
$$

where $\gamma_{i}(i=1,2,3)$ is the solution to $(50 \mathrm{a})-(50 \mathrm{c})$ with $e(\cdot)$ replaced by 0 , and

$$
\begin{gathered}
\widehat{x}(t)=\Upsilon_{0}^{t} x_{0}+\int_{0}^{t} \Upsilon_{s}^{t}\left[f_{1}(s) d s+g_{1}(s) d W_{1}(s)\right. \\
\left.+g_{2}(s) d W_{2}(s)\right]
\end{gathered}
$$

with

$$
\begin{gathered}
\Upsilon_{s}^{t}=\exp \left\{\int_{s}^{t}\left[a(r)-b_{1}^{2}(r) m_{1}^{-1}(r) \gamma_{1}(r)\right] d r\right\}, \\
f_{1}=-\left(b_{2}^{2} m_{2}^{-1} \alpha_{2}+b_{1}^{2} m_{1}^{-1} \gamma_{2}\right) \mathbb{E} x-b_{1}^{2} m_{1}^{-1} \gamma_{3}-b_{2}^{2} m_{2}^{-1} \beta_{2}+c .
\end{gathered}
$$

The relation between $\check{y}_{2}$ and $(\check{x}, \mathbb{E} x)$ is as follows:

$$
\bar{y}_{2}=\tau_{1} \check{x}+\tau_{2} \mathbb{E} x+\tau_{3},
$$

where $\tau_{i}(i=1,2,3)$ is the unique solution to $(56 \mathrm{a})-(56 \mathrm{c})$ with $e(\cdot)$ replaced by 0 , and

$$
\check{x}(t)=\Psi_{0}^{t} x_{0}+\int_{0}^{t} \Psi_{s}^{t}\left[f_{2}(s) d s+g_{3}(s) d W_{3}(s)\right],
$$

with

$$
\begin{gathered}
\Psi_{s}^{t}=\exp \left\{\int_{s}^{t}\left[a(r)-b_{2}^{2}(r) m_{2}^{-1}(r) \tau_{1}(r)\right] d r\right\}, \\
f_{2}=-\left(b_{2}^{2} m_{2}^{-1} \tau_{2}+b_{1}^{2} m_{1}^{-1} \alpha_{1}\right) \mathbb{E} x-b_{1}^{2} m_{1}^{-1} \beta_{1}-b_{2}^{2} m_{2}^{-1} \tau_{3}+c .
\end{gathered}
$$

Then Theorem 21 can be rewritten as follows.

Theorem 22. Under the assumption $\left(\mathrm{H}_{3}\right)$ and $\left(\mathrm{H}_{4}\right)$, Problem (LQ NZSDG) has a unique Nash equilibrium point denoted by

$$
\begin{aligned}
& u_{1}(t)=-m_{1}^{-1}(t) b_{1}(t)\left(\gamma_{1}(t) \hat{x}(t)+\gamma_{2}(t) \mathbb{E} x(t)+\gamma_{3}(t)\right), \\
& u_{2}(t)=-m_{2}^{-1}(t) b_{2}(t)\left(\tau_{1}(t) \check{x}(t)+\tau_{2}(t) \mathbb{E} x(t)+\tau_{3}(t)\right),
\end{aligned}
$$

where $\mathbb{E} x, \widehat{x}$, and $\check{x}$ are shown in (77), (79), and (82), respectively, and $\gamma_{i}$ and $\tau_{i}(i=1,2,3)$ are uniquely determined by the systems of $(50 \mathrm{a})-(50 \mathrm{c})$ and $(56 \mathrm{a})-(56 \mathrm{c})$ with $e(\cdot)$ replaced by 0 , respectively.

Remark 23. In the cases similar to Case 4 , such as $\mathscr{G}_{t}^{1}=\mathscr{F}_{t}^{1,3}$ and $\mathscr{G}_{t}^{2}=\mathscr{F}_{t}^{2}, \mathscr{G}_{t}^{1}=\mathscr{F}_{t}^{2,3}$ and $\mathscr{G}_{t}^{2}=\mathscr{F}_{t}^{1}$, the corresponding results can be easily derived.

\section{Conclusion Remark}

In this paper, we investigate LQ nonzero sum differential game problem where the information available to players is asymmetric. We discuss the game problem under the four classes of cases: (i) $\mathscr{G}_{t}^{1}=\mathscr{F}_{t}^{1,2}$ and $\mathscr{G}_{t}^{2}=\mathscr{F}_{t}^{2,3}$; (ii) $\mathscr{G}_{t}^{1}=\mathscr{F}_{t}^{1,2}$ and $\mathscr{G}_{t}^{2}=\mathscr{F}_{t}^{2}$; (iii) $\mathscr{G}_{t}^{1}=\mathscr{F}_{t}$ and $\mathscr{G}_{t}^{2}=\mathscr{F}_{t}^{2}$; (iv) $\mathscr{G}_{t}^{1}=\mathscr{F}_{t}^{1,2}$ and $\mathscr{G}_{t}^{2}=\mathscr{F}_{t}^{3}$. Some forward-backward stochastic filtering 
equations with respect to the asymmetric information $\mathscr{G}_{t}^{1}$ and $\mathscr{G}_{t}^{2}$ are introduced and the existence and uniqueness of the solutions are proved. Finally, the corresponding unique Nash equilibrium point is represented in a feedback form of the optimal filtering of the state, through the solutions of some Riccati equations.

\section{Conflict of Interests}

The authors declare that there is no conflict of interests regarding the publication of this paper.

\section{Acknolwedgments}

Hua Xiao acknowledges the financial support from the National Nature Science Foundation of China (11201263, 11371228), the Program for New Century Excellent Talents in University of China (NCET-12-0338), and the Postdoctor Foundation of China (2013M540540) and the Nature Science Foundation of Shandong Province (ZR2012AQ004, BS2011SF010). Dejian Chang acknowledges the financial support from the National Nature Science Foundation of China (10921101, 61174092) and the National Nature Science Foundation for Distinguished Young Scholars of China (11125102).

\section{References}

[1] G. Gennotte, "Optimal portfolio choice under incomplete information," Journal of Finance, vol. 41, pp. 733-746, 1986.

[2] J. Xiong and X. Y. Zhou, "Mean-variance portfolio selection under partial information," SIAM Journal on Control and Optimization, vol. 46, no. 1, pp. 156-175, 2007.

[3] F. Biagini and B. Øksendal, "Minimal variance hedging for insider trading," International Journal of Theoretical and Applied Finance, vol. 9, no. 8, pp. 1351-1375, 2006.

[4] L. Campi, "Some results on quadratic hedging with insider trading," Stochastics, vol. 77, no. 4, pp. 327-348, 2005.

[5] H. M. Müller, "The first-best sharing rule in the continuoustime principal-agent problem with exponential utility," Journal of Economic Theory, vol. 79, no. 2, pp. 276-280, 1998.

[6] H. M. Müller, "Asymptotic efficiency in dynamic principalagent problems," Journal of Economic Theory, vol. 91, no. 2, pp. 292-301, 2000.

[7] S. Hamadène, "Nonzero sum linear-quadratic stochastic differential games and backward-forward equations," Stochastic Analysis and Applications, vol. 17, no. 1, pp. 117-130, 1999.

[8] Q. Meng, "General linear quadratic optimal stochastic contr ol problem driven by a Brownian motion and a poisson random martingale measure with random coefficients," Stochastic Analysis and Applications, vol. 32, no. 1, pp. 88-109, 2014.

[9] L. Mou and J. Yong, "Two-person zero-sum linear quadratic stochastic differential games by a Hilbert space method," Journal of Industrial and Management Optimization, vol. 2, no. 1, pp. 95$117,2006$.

[10] S. Tang, "General linear quadratic optimal stochastic control problems with random coefficients: linear stochastic hamilton systems and backward stochastic riccati equations," SIAM Journal on Control and Optimization, vol. 42, no. 1, pp. 53-75, 2003.
[11] Z. Wu and Z.-Y. Yu, "Linear quadratic nonzero-sum differential games with random jumps," Applied Mathematics and Mechanics, vol. 26, no. 8, pp. 1034-1039, 2005.

[12] J. Yong, "A leader-follower stochastic linear quadratic differential game," SIAM Journal on Control and Optimization, vol. 41, no. 4, pp. 1015-1041, 2002.

[13] Z. Yu, "Linear-quadratic optimal control and nonzero-sum differential game of forward-backward stochastic system," Asian Journal of Control, vol. 14, no. 1, pp. 173-185, 2012.

[14] Y. Hu and B. Øksendal, "Partial information linear quadratic control for jump diffusions," SIAM Journal on Control and Optimization, vol. 47, no. 4, pp. 1744-1761, 2008.

[15] K. L. Chung, A Course in Probability Theory, Academic Press, San Diego, Calif, USA, 3rd edition, 2001.

[16] S. Tang, "The maximum principle for partially observed optimal control of stochastic differential equations," SIAM Journal on Control and Optimization, vol. 36, no. 5, pp. 1596-1617, 1998.

[17] F. Baghery and B. Øksendal, "A maximum principle for stochastic control with partial information," Stochastic Analysis and Applications, vol. 25, no. 3, pp. 705-717, 2007.

[18] J. Xiong, An Introduction to Stochastic Filtering Theory, Oxford University Press, Oxford, UK, 2008.

[19] J. Huang, G. Wang, and J. Xiong, "A maximum principle for partial information backward stochastic control problems with applications," SIAM Journal on Control and Optimization, vol. 48, no. 4, pp. 2106-2117, 2009.

[20] E. Hui and H. Xiao, "Differential games of partial information forwardbackward doubly stochastic differential equations and applications," ESAIM: Control, Optimisation and Calculus of Variations, vol. 20, no. 1, pp. 78-94, 2014.

[21] G. Wang, Z. Wu, and J. Xiong, "Maximum principles for forward-backward stochastic control systems with correlated state and observation noises," SIAM Journal on Control and Optimization, vol. 51, no. 1, pp. 491-524, 2013.

[22] G. Wang and Z. Yu, "A partial information non-zero sum differential game of backward stochastic differential equations with applications," Automatica A: Journal of IFAC, vol. 48, no. 2, pp. 342-352, 2012.

[23] G. Wang, C. Zhang, and W. Zhang, "Stochastic maximum principle for mean-field type optimal control under partial information," IEEE Transactions on Automatic Control, vol. 59, no. 2, pp. 522-528, 2014.

[24] H. Xiao, "The maximum principle for partially observed optimal control of forward-backward stochastic systems with random jumps," Journal of Systems Science and Complexity, vol. 24, no. 6, pp. 1083-1099, 2011.

[25] H. Xiao and G. Wang, "The filtering equations of forwardbackward stochastic systems with random jumps and applications to partial information stochastic optimal control," Stochastic Analysis and Applications, vol. 28, no. 6, pp. 1003-1019, 2010.

[26] H. Xiao and G. Wang, "A necessary condition for optimal control of initial coupled forward-backward stochastic differential equations with partial information," Journal of Applied Mathematics and Computing, vol. 37, no. 1-2, pp. 347-359, 2011.

[27] E. Pardoux and S. G. Peng, "Adapted solution of a backward stochastic differential equation," Systems \& Control Letters, vol. 14 , no. 1, pp. 55-61, 1990. 


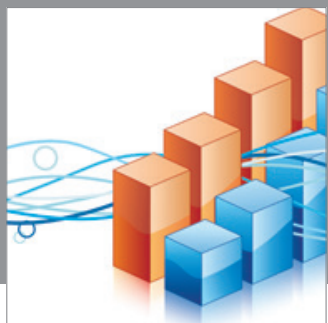

Advances in

Operations Research

mansans

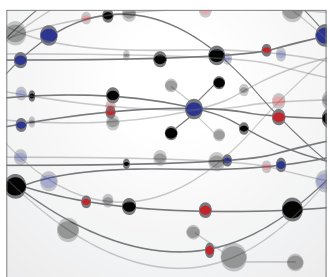

The Scientific World Journal
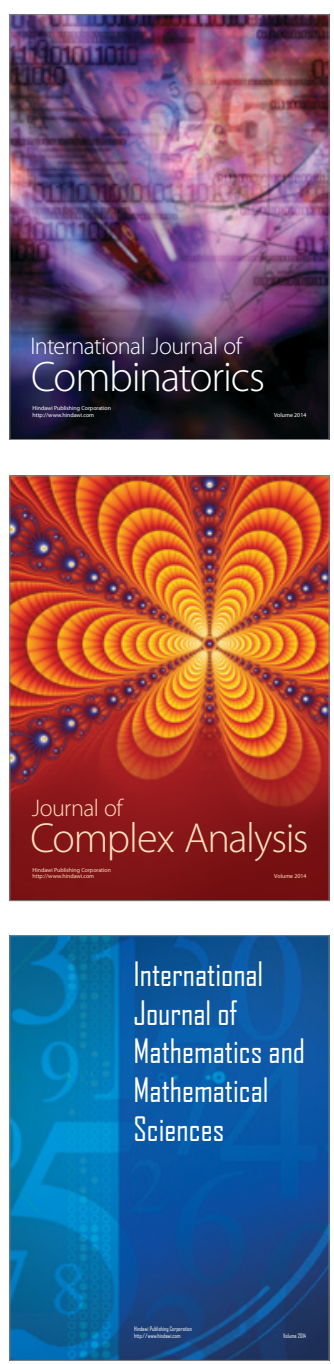
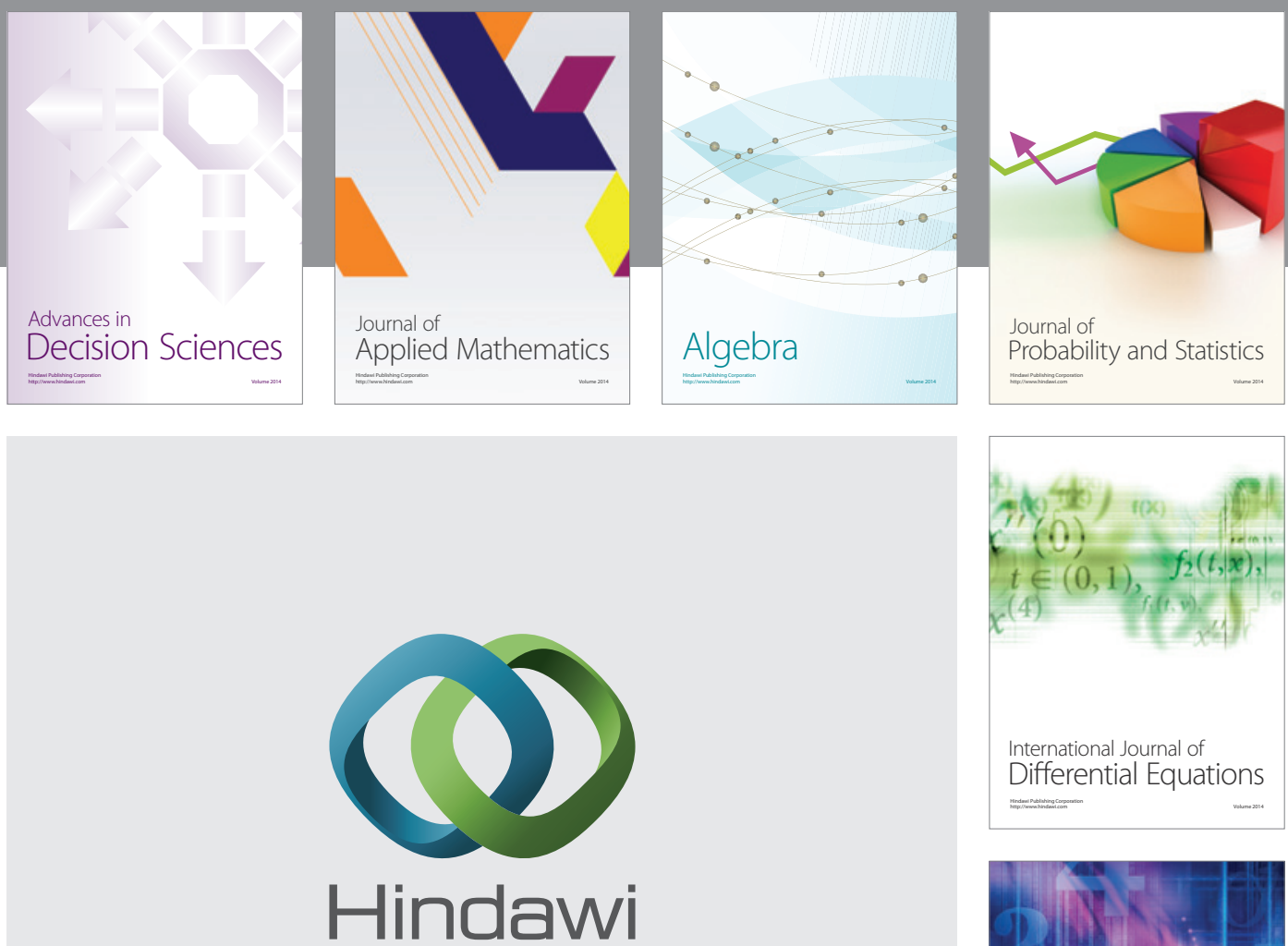

Submit your manuscripts at http://www.hindawi.com
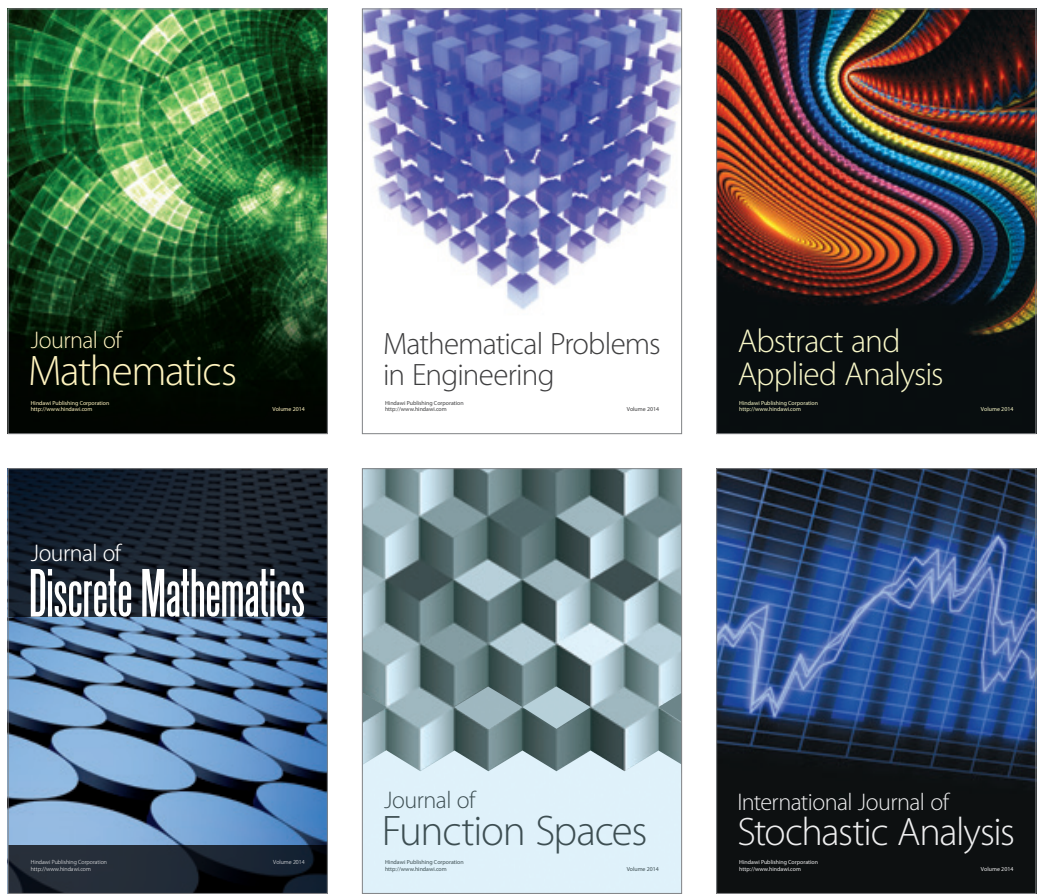

Journal of

Function Spaces

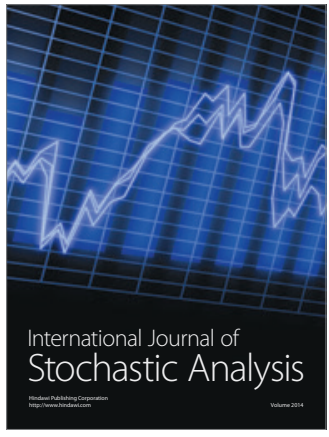

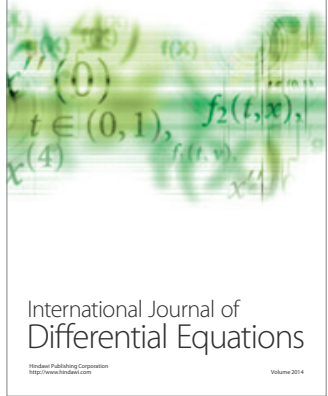
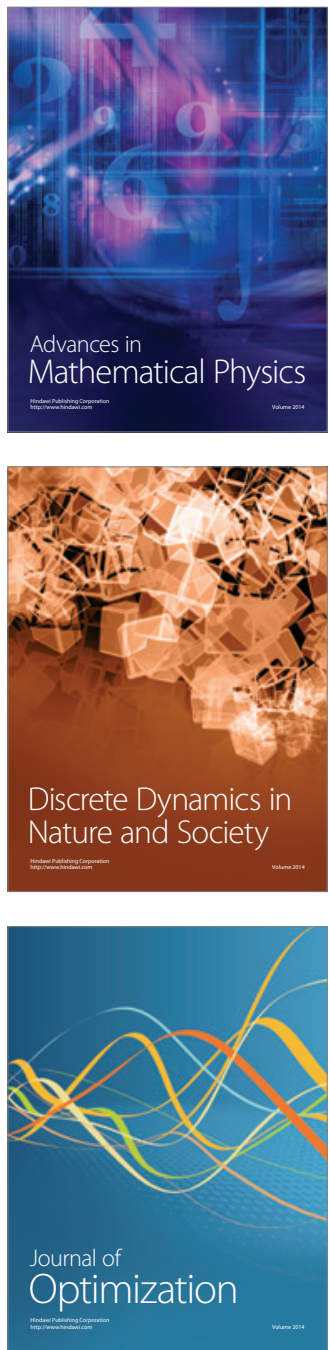